\title{
Anchoring of single-platinum-adatoms on cyanographene: Experiment and theory
}

\author{
Rostislav Langer ${ }^{\mathrm{a}, 1}$, Edvin Fako $^{\mathrm{c}, 1}$, Piotr Błoński ${ }^{\mathrm{b}, *}$, Miroslav Vavrečka ${ }^{\mathrm{a}, \mathrm{b}}$, Aristides Bakandritsos ${ }^{\mathrm{b}}$, \\ Michal Otyepka ${ }^{\mathrm{a}, \mathrm{b}}$, Núria López ${ }^{\mathrm{c}, *}$ \\ a Department of Physical Chemistry, Faculty of Science, Palacký University, Olomouc, Czech Republic \\ ${ }^{\mathrm{b}}$ Regional Centre of Advanced Technologies and Materials, Faculty of Science, Palacký University, Olomouc, Czech Republic \\ ${ }^{\mathrm{c}}$ Institute of Chemical Research of Catalonia (ICIQ), The Barcelona Institute of Science and Technology, Tarragona, Spain
}

\begin{abstract}
A B S T R A C T
Graphene decorated with isolated single atoms (SAs) offers new vista to magnetic and spintronic devices up to single-atom catalysts. While $s p$ atoms can be efficiently bound to graphene, $d$-block atoms require anchoring groups to prevent nanoparticle formation. Identification of suitable binding sites is a challenging task because the interaction among graphene, anchoring groups and adatoms is very complex. Using density functional theory (DFT) we explored strength and nature of interactions of graphene covalently functionalized by $-\mathrm{OH},-\mathrm{CN},-\mathrm{F}$, and $-\mathrm{H}$ groups as anchors for Pt SAs. Both theory and experiment showed that $-\mathrm{CN}$ groups acted as suitable ligand enabling immobilization of $3.7 \mathrm{wt} \%$ single Pt adatoms. The findings imply that $\mathrm{CN}$ functionalized graphene, i.e., cyanographene, is a perspective material for anchoring metal adatoms with potential implications as single-atom-catalysts.
\end{abstract}

\section{Introduction}

Since the discovery of graphene [1-3], its vast application potential has driven research in many fields [4-6]. Graphene is one of the most studied materials of recent decades making it a popular model system for graphitic materials [7]. Many of the fascinating properties of pristine graphene can be tuned by functionalization [8-11] and put to use in diverse applications, including electrical devices, sensors, spintronics and other [12-17].

High specific surface and affordable price make graphene an attractive support for heterogeneous catalysis. Atomistic design of the active phase is vital for unlocking the full potential of heterogeneously catalyzed industrial (and other) chemical reactions [18]. These catalytic reactions take place on (typically) metal surfaces and therefore bulk metal atoms that are not accessible by the reactants are not involved in the catalysis. There are clear benefits to be had from reducing the amount of such inactive atoms. The downsizing of heterogeneous metal catalysts is a prominent way of improving their catalytic properties, and single atom catalysts (SACs) are at the border of what is possible in terms of advanc-

\footnotetext{
* Corresponding authors.

E-mail addresses: piotr.blonski@upol.cz (P. Błoński), nlopez@iciq.es (N. López).

1 These authors are contributed equally to this work.
}

ing this trend [19-21]. The benefit of SACs lies not only in that they provide the highest possible surface to volume ratio of any heterogeneous catalyst, but they also give rise to several specific interactions enhancing selectivity. SACs are at the ultimate frontier in atom economy with nearly $100 \%$ metal loading being utilized to form accessible active sites. Moreover, the well-defined structure and strong interaction between the support and SAC suggest easy reusability and recycling of the catalytic material [22,23].

Particularly, carbon supported SACs have recently been successfully employed for several processes traditionally considered to be difficult to perform utilizing a heterogeneous catalyst, e.g. Pd-catalyzed coupling reactions or Pd-SAs enhanced catalytic reactivity in the semi-hydrogenation of acetylene to ethylene [24-27]. Despite the successful application of SACs for an increasing number of applications, only a few highly stable coordination environments [28-32] have yet been experimentally characterized. Graphene derivatives were identified as promising supports for single adatoms $[33,34]$, however, stabilizing the individual metal atoms on the support remains a significant challenge in further development of efficient and stable SACs. The doping of graphene with $s p$ elements (particularly $s p^{3}$ doping) unlocked the means of adjusting the electronic structure of graphene [16], however, the relatively low interaction of the pristine graphene layer with the $d$-block adatoms [35], implies that a different approach must be utilized when trying to stabilize these isolated atoms on graphene 
[36-38]. Binding isolated metal atoms to already functionalized graphene, provides additional interaction between the metal and functional group with respect to pristine graphene and may represent an efficient way for synthesis of new branch of graphene-based derivatives applicable in electronics and single atom catalysis. To advance this field, it is necessary to identify suitable anchoring groups for metal single atoms and deeply understand the nature of metal-linker-graphene interaction and influence of the adatom on the electronic structure of the graphene-derivative.

Here, we discuss the possibility of attaching single platinum adatoms to diluted graphene derivatives, because platinum is often used as the active center in various important catalytic processes $[25,32,39,40]$. We considered existing graphene derivatives, i.e., fluorinated graphene [41], graphene oxide [42], cyanographene [43] and graphane [44], i.e., derivatives containing functional groups $(\mathrm{X}=-\mathrm{F},-\mathrm{OH},-\mathrm{CN}$, and $-\mathrm{H})$, which can potentially anchor the platinum single atoms (Fig. S1 and Table S1). Using theoretical calculations we identified the nitrile group as the most efficient anchor for the Pt adatoms (in $\mathrm{Pt}^{0}$ and $\mathrm{Pt}^{\mathrm{II}}$ oxidation states, Fig. S2) which otherwise easily diffuse along the pristine graphene surface (Fig. S3 and Table S2). We also successfully grafted the Pt single atoms to cyanographene with high content, $3.7 \mathrm{wt} \%$. It should be noted, that the cyanographene was very recently used for anchoring the $\mathrm{Cu}$ atoms and applied as SAC for oxidative amine coupling and alcohol oxidation reactions [21]. All these results show that the nitrile groups are well suited for anchoring of metal single atoms to graphene and identify cyanographene an ideal platform for synthesis of a wide portfolio of SACs.

\section{Materials and methods}

\subsection{Computational details}

The first-principle spin-polarized density functional theory (DFT) calculations were performed using the Vienna Ab initio Simulation Package (VASP) code $[45,46]$. The Perdew-Burke-Ernzerhof functional (PBE) [47] was employed with inner electrons represented as projector augmented waves (PAW) $[48,49]$. Dispersion contributions were introduced through the D3 approach [50,51]. The valence monoelectronic states expanded in plane waves with a cut-off kinetic energy of $500 \mathrm{eV}$. The Brillouin zone was sampled with a $6 \times 6 \times 1$ (structure and cell optimization) and $15 \times 15 \times 1$ (DOS and energy calculations) $\Gamma$-centered grid. Graphene was modelled as a $(3 \times 3)$ and $(6 \times 6)$ supercell containing 18 and 72 carbon atoms. The molecules were placed in a triclinic box with $18 \AA$ sides. We considered diluted systems containing one functional group attached on-top of the graphene lattice and systems with two functional groups in the ortho/trans position (Fig. S1). In all cases the ionic and electronic convergence criteria were $10^{-3} \mathrm{eV}$ and $10^{-6} \mathrm{eV}$, respectively, in the self-consistent field (SCF) cycle. A dipole correction was employed along the z-direction [52]. Transition states were located following the climbing image nudged elastic band procedure (CI-NEB) [53,54]. Analysis of the charges was performed using the Bader approach [55]. Total magnetic moments were calculated as the difference between the numbers of electrons in occupied majority- and minority-spin states.

The stability of $\mathrm{g}-\mathrm{X}$ structures (X: fluorine $-\mathrm{F}$, hydroxyl $-\mathrm{OH}$, nitrile $-\mathrm{CN}$, hydrogen $-\mathrm{H}$ or platinum $\mathrm{Pt}$ ) shown in Fig. S1 was assessed in terms of the binding energy per group

$E_{\text {bind }}=\left(E_{g-X}-E_{g}-n \cdot E_{X}\right) / n$

where $E_{g-X}, E_{g}$, and $E_{X}$ denote the total energy of the functionalized graphene, pristine graphene, and functional groups, $n$ stands for number of functional groups. The average $\mathrm{C}-\mathrm{C}$ bond distance in pristine graphene $(1.43 \AA$ ) agrees well with experimental results
[56]. A functional group has been placed onto three high symmetry sites of graphene lattice, i.e. top (t), bridge (b) and six-fold hollow (h) position. The thermodynamic stability of all reported g-X-Pt configurations was analysed in terms of the binding energy per platinum adatom

$E_{\text {bind } / P t}=E_{g-X-P t}-E_{g-X}-E_{P t}$

where $E_{g-X-P t}, E_{g-X}$, and $E_{P t}$ and denote the total energy of functionalized graphene doped by Pt adatom, functionalized graphene and Pt adatom, respectively.

The finite model calculations of g- $(\mathrm{CN})_{2}-\mathrm{Pt}$ (Fig. S2) were performed using Gaussian software [57] employing PBE exchangecorrelation functional [47,58] and empirical dispersions D3 [51]. All structures can be retrieved from the ioChem-BD database [59] following the link [https://doi.org/10.19061/iochem-bd-1-101].

\subsection{Materials}

Cyanographene (g-CN) was synthesized according to the previously reported procedure [43], $\mathrm{K}_{2}\left[\mathrm{PtCl}_{4}\right]$ was purchased from Sigma-Aldrich, ethanol absolute from Penta, ultrapure water $\left(18 \mathrm{M} \Omega \mathrm{cm}^{-1}\right)$ was used in all cases, produced from a Puris Esse-UP Ultra Pure Water System.

\subsection{Synthesis of the cyanographene/Pt(II) hybrid (g-CN/Pt)}

g-CN (120 mg in $\left.12 \mathrm{~mL} \mathrm{H}_{2} \mathrm{O}\right)$ and $\mathrm{K}_{2}\left[\mathrm{PtCl}_{4}\right](162 \mathrm{mg}$ in $12 \mathrm{~mL}$ $\mathrm{H}_{2} \mathrm{O}$ ) were mixed and microwave-heated at $60^{\circ} \mathrm{C}$ for $15 \mathrm{~min}$. (850 W, 300 Anthon par microwave synthesizer). Then, the mixture was centrifuged and washed three times with water and once with ethanol.

\subsection{Experimental techniques}

The samples were dispersed in water, sonicated for $5 \mathrm{~min}$ and one drop put on copper grid with Holey carbon film. After drying on the air at room temperature, the samples were measured by High Resolution Transmission Electron Microscope (HR-TEM) Titan G2 (FEI) with Image corrector on accelerating voltage $80 \mathrm{kV}$. Images were taken with BM UltraScan CCD camera (Gatan). Energy Dispersive Spectrometry (EDS) was performed in Scanning TEM (STEM) mode by Super-X system with four silicon drift detectors (Bruker). STEM images were taken with HAADF detector 3000 (Fishione).

The concentration of metals was determined by the atomic absorption spectroscopy (AAS) technique, using a graphite furnace (ContrAA 600; Analytik Jena AG, Germany) equipped with a highresolution Echelle double monochromator (spectral band width, $2 \mathrm{pm}$ at $200 \mathrm{~nm}$ ). A xenon lamp was used as a continuum radiation source.

High-resolution X-ray photoelectron spectroscopy (HR-XPS) was performed using a PHI VersaProbe II spectrometer (Physical Electronics) equipped with an $\mathrm{Al} \mathrm{K \alpha}$ source $(15 \mathrm{kV}, 50 \mathrm{~W})$. All spectra were acquired at room temperature $\left(22^{\circ} \mathrm{C}\right)$, under vacuum $\left(1.4 \times 10^{-7} \mathrm{~Pa}\right)$. High-resolution spectra were acquired by setting the pass energy to $23.500 \mathrm{eV}$ and the step size to $0.200 \mathrm{eV}$. These spectra were analyzed using the MultiPak (Ulvac-PHI, Inc.) program. The binding energy values were reported with respect to the C1s lowest peak energy at $284.70 \mathrm{eV}$.

\section{Results and discussions}

The bond strengths of functional groups to graphene depend on the hybrid state of the neighboring carbon atoms. Generally, the bonds are weak if the $s p^{3}$ carbon bearing the functional group is attached to three $\mathrm{sp}^{2}$ carbon atoms, however, their strength 

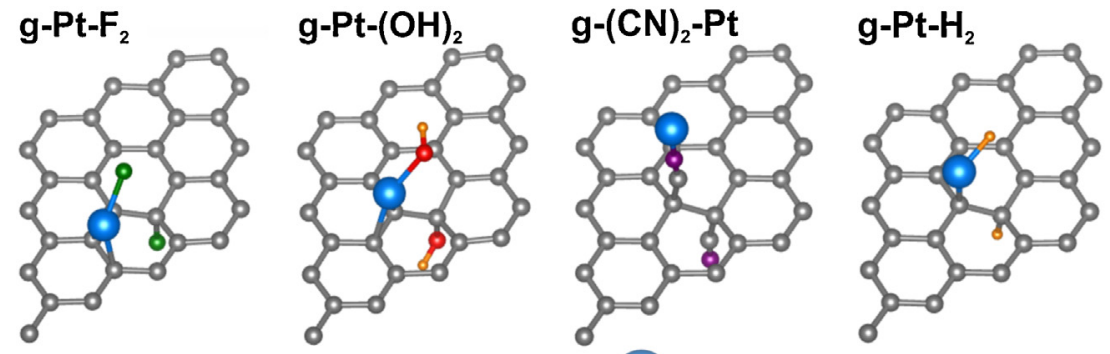

$0 \mathrm{CONHPt}$

Fig. 1. The most stable structures of g-X-Pt complexes: two-sided fluorinated g-Pt- $\mathrm{F}_{2}$, hydroxylated g-Pt- $(\mathrm{OH})_{2}$, nitrilated g- $(\mathrm{CN})_{2}-\mathrm{Pt}$ and hydrogenated g-Pt- $\mathrm{H}_{2}$.
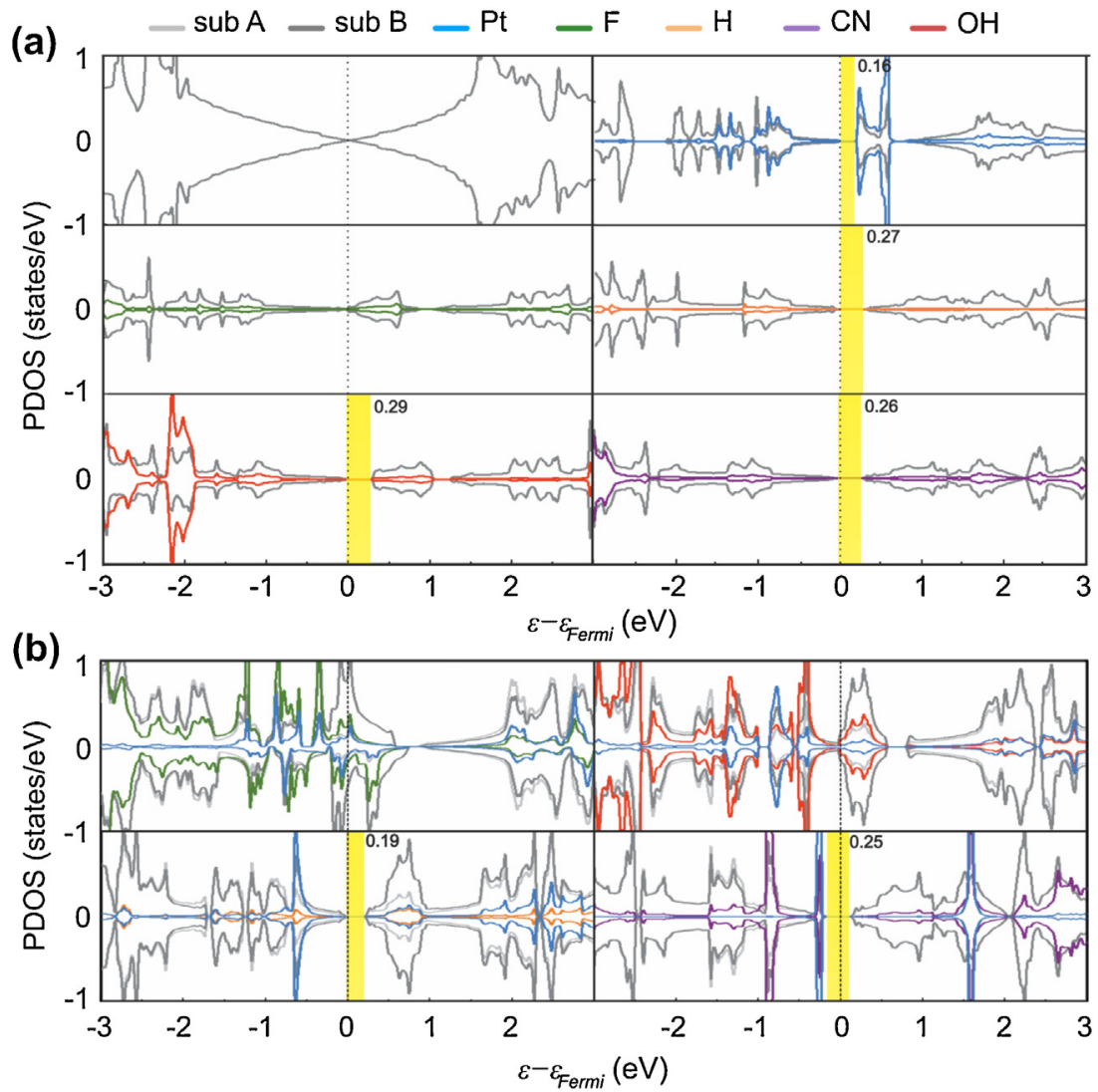

Fig. 2. Spin-polarized PDOS plots of two-sided functionalized graphene (a) g- $\mathrm{X}_{2}$, (b) g- $\mathrm{X}_{2}$-Pt. The Fermi level is set to zero. Band-gaps are depicted by vertical bars; numbers correspond to the width of the band-gap (in eV).

increase if the $s p^{3}$ carbon is attached to another $s p^{3}$ (bearing an attached group in trans-position) and just two $\mathrm{sp}^{2}$ carbon atoms (see Section 2.1 in Supporting Information for details). Therefore, we have focused on the double-sided functionalized graphene $\mathrm{g}-\mathrm{X}_{2}$ with functional groups in ortho/trans positions (see Section 2.2 in Supporting Information for details) and the results concerning onesided functionalized graphene $\mathrm{g}-\mathrm{X}$ are shown in Section 2.3 and 2.4 in the Supporting Information.

We considered all unique positions of Pt atoms relative to the functional groups (Fig. S4). Surprisingly, the Pt adatom cleaved the bonds of $-\mathrm{F},-\mathrm{OH}$, and $-\mathrm{H}$ to graphene and they released fluorine, hydroxyl, and hydrogen groups attached on-top of the Pt adatom, forming graphene- $\mathrm{Pt}-\mathrm{F}(-\mathrm{OH}$, or $-\mathrm{H})$ complexes (Fig. 1). This process was exothermic with binding energies per platinum adatom from -2.79 to $-4.05 \mathrm{eV}$ (Eq. 2, Table S3, and Fig. S5). An easy diffusion of -Pt-X moiety following a top-bridge path was enabled by low diffusion barriers (0.13-0.44 eV, Table S4) similar to the diffusion barrier of isolated platinum adatom $(0.15 \mathrm{eV}$, Table S2). This finding agrees with the experimental observation that $-\mathrm{OH}$ groups were released from graphene after the Pt deposition [60,61]. On the other hand, Pt adatom did not cause the release of $-\mathrm{CN}$ from the graphene surface and is instead coordinated on top of the $-\mathrm{CN}$ group, forming a linear g-CN-Pt complex (Fig. 1). The anchoring of Pt adatom above or in the vicinity of the $-\mathrm{CN}$ group (Fig. S5c1-4) prevented any potential detrimental processes, e.g. agglomeration, diffusion or leaching, with a high top-bridge diffusion barrier of $1.34 \mathrm{eV}$ (Table S4). Such high diffusion barriers reveal that thermal effects do not compromise the long term stability of this material. The g-CN based catalysts remained highly stabile even upon recycling [21,62]. Additionally, when a Pt adatom is bound on top of a -CN group, this leaves the coordination sphere of Pt accessible which is desirable for catalytic activity. One shall note that both the release of $-\mathrm{F},-\mathrm{OH}$, and $-\mathrm{H}$ from the graphene lattice to 
(a)

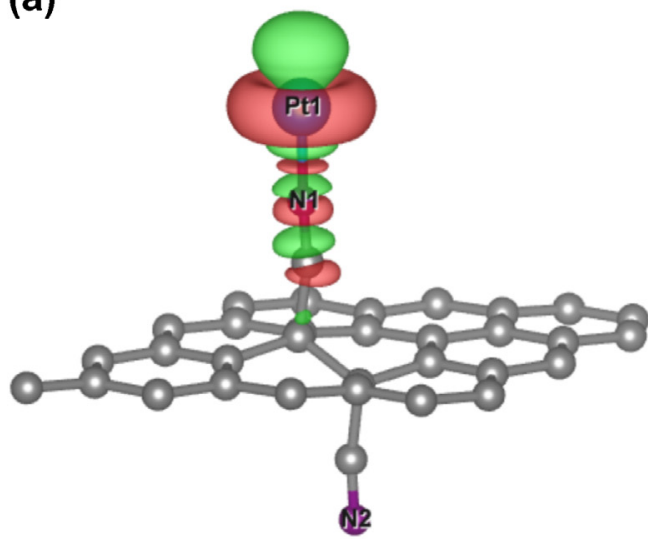

(b)

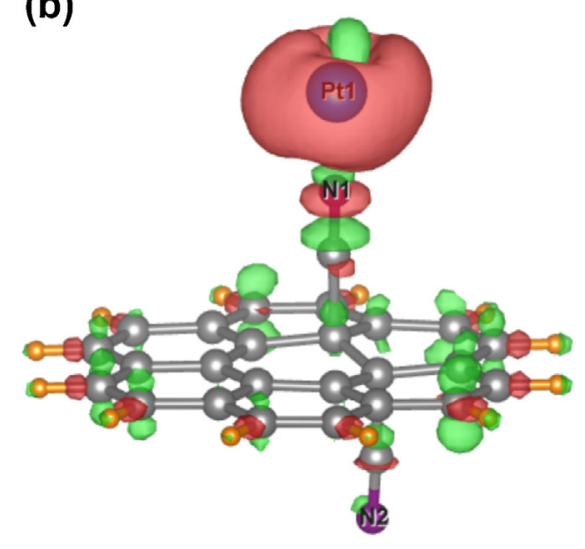

Fig. 3. (a) Charge density difference plot of periodic model g-CN $\mathrm{CN}_{2}-\mathrm{Pt}$ (isovalue 5 me $^{-3}$ ) and (b) charge density difference plot of finite model g-CN 2 -Pt $\mathrm{t}^{\mathrm{II}}$ in gas phase (isovalue $\left.5 \mathrm{me} \AA^{-3}\right)$.
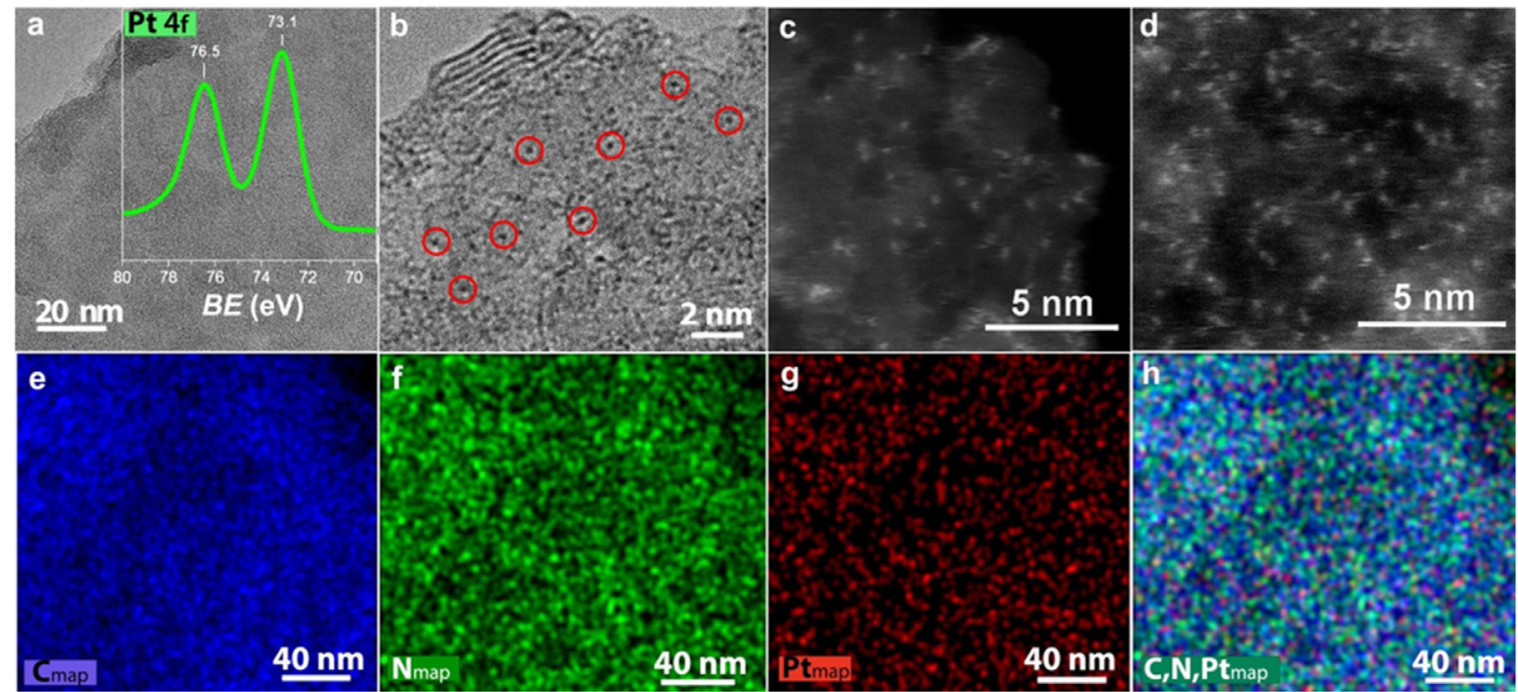

Fig. 4. Representative HR-TEM image of a flake from the g-CN/Pt hybrid and HR-XPS of the Pt $4 \mathrm{f}$ region (a), higher magnification HR-TEM image showing high-contrast spots probably originating from embedded Pt(II) ions (b), HAADF-STEM images showing the heavier (and thus brighter) single metal ions embedded in the $\mathrm{g}$ - CN support (c,d) and EDS elemental mapping performed on g-CN/Pt for carbon (e), nitrogen (f), platinum (g), and combined C, $\mathrm{N}$ and $\mathrm{Pt}(\mathrm{h})$.

form g-Pt-F( $\left.\mathrm{F}_{2}\right)$, g-Pt- $\mathrm{OH}\left(\mathrm{OH}_{2}\right)$, and g-Pt- $\mathrm{H}\left(\mathrm{H}_{2}\right)$ complexes and the formation of $\mathrm{g}-\mathrm{CN}\left(\mathrm{CN}_{2}\right)-\mathrm{Pt}$ was predicted for one-sided and two-sided functionalized graphene (Tables S5-S16).

The chemical functionalization of graphene, which is a nonmagnetic zero bandgap semi-metal [2] with its valence and conductive bands crossing at the Dirac point at the Fermi level $\left(E_{F}\right)$, significantly changes its electronic structure. The covalent bonding of $-\mathrm{H},-\mathrm{OH}$, and $-\mathrm{CN}$ to the graphene lattice in trans-positions opened the graphene's zero-band-gap, while fluorinated graphene is a p-type metal (Fig. 2a and Fig. S6-S9, Table S3 and Tables S5S8) [63-65]. All $\mathrm{g}-\mathrm{X}_{2}$ systems are non-magnetic. The deposition of Pt adatom onto the fluorinated graphene leading to the formation of graphene-Pt- $\mathrm{F}_{2}$ complex, further strengthen the metallic character of the system (Fig. 2b, Fig. S10). Also, the electronic gap at $E_{F}$ for the graphene- $(\mathrm{OH})_{2}$ system was closed by the formation of the g-Pt- $(\mathrm{OH})_{2}$ complex (Fig. 2b and Fig. S11) although with a much lower DOS at $E_{F}$. Accordingly, the DOS of the latter system is spin symmetric, whereas the $\mathrm{g}-\mathrm{Pt}-\mathrm{F}_{2}$ complex is spin-polarized. The formation of graphene-Pt- $\mathrm{H}_{2}$ complex lower the electronic gap of the hydrogenated graphene from 0.27 to $0.19 \mathrm{eV}$, while the electronic gap of cyanographene $(0.26 \mathrm{eV})$ was hardly modified $(0.25 \mathrm{eV})$ due to the presence of Pt bound on top of the $-\mathrm{CN}$ group
(Fig. 2b and Fig. S12-S13). The conductive character of the material can be highly advantageous in electrochemical sensing, capacitors, (photo)redox and (photo)electrocatalytic applications due to the possibility of storing and shuttling electrons $[17,21,43,62,66]$. Both g-Pt- $\mathrm{H}_{2}$ and $\mathrm{g}-(\mathrm{CN})_{2}-\mathrm{Pt}$ are non-magnetic with spin symmetric DOSs. Whereas for the metallic $\mathrm{g}-\mathrm{Pt}-\mathrm{F}_{2}$ and $\mathrm{g}-\mathrm{Pt}-(\mathrm{OH})_{2}$ complexes the charge depletion on Pt was the greatest (respectively $0.47 e$ and $0.34 e$ ), the charge lost on Pt atom bound on-top of $-\mathrm{CN}$ was only $0.06 e$, and in the g-Pt- $\mathrm{H}_{2}$ complex there was hardly any chargetransfer from/to Pt observed (Table S3). Fig. 3 and Fig. S14 show the difference-electron densities calculated for the g-CN-Pt complex with platinum in both oxidation $\mathrm{Pt}^{0}$ and $\mathrm{Pt}^{\mathrm{II}}$. The picture demonstrates a polar-covalent bond formation between Pt and $\mathrm{N}\left(E_{\text {bind }} / \mathrm{Pt}\right.$ $=-2.29 \mathrm{eV}$ ) associated with the electron transfer to antibonding $\sigma_{d}{ }^{*}$ state of the Pt atom, which is accompanied with the accumulation of electrons in bonding states between Pt, N, and C atoms, shown as a charge depletion both on the Pt site within the donut-shaped ring parallel to the graphene plane, and on the $\mathrm{N}$ site.

Both platinum atom $\mathrm{Pt}^{0}$ and $\mathrm{Pt}^{\mathrm{II}}$ ion which represents a common oxidation state of platinum were considered in the gas phase and water environment using an implicit solvent model. As expected, the calculations (Eq. 2) identified that $\mathrm{Pt}^{\mathrm{II}}$ binds to g-CN more 
strongly $(13.92 \mathrm{eV})$ than $\mathrm{Pt}^{0}(2.35 \mathrm{eV})$ in vacuum. In water, the interaction of $\mathrm{Pt}^{\mathrm{II}}$ to $\mathrm{g}-\mathrm{CN}$ significantly dropped to $3.98 \mathrm{eV}$, while the interaction of $\mathrm{Pt}^{0}$ remained less affected amounting to $2.63 \mathrm{eV}$. These calculations suggest that the nitrile group is well suited for anchoring platinum in both $\mathrm{Pt}^{0}$ and $\mathrm{Pt}^{\mathrm{II}}$ states (Table $\mathrm{S} 17$ and $\mathrm{S} 18$ ).

The ability of g-CN to bind Pt atoms was evaluated experimentally by mixing of aqueous dispersions of the cyanographene with the $\mathrm{Pt}^{\mathrm{II}}$ salt. The obtained g-CN/Pt hybrid was analyzed with HRTEM, revealing the absence of any inorganic crystalline phases, i.e. nanoparticles (Fig. 4a, Fig. S29), but unveiled atomic sized high-contrast spots, suggesting the presence of heavy metal atoms (Fig. 4b). This was confirmed by the abundant bright spots from metal atoms, revealed via the sub-Ångstrom resolution aberration corrected high-angle annular dark-field (HAADF) STEM in Fig. 4c,d. Furthermore, Pt atoms appear to populate densely and homogeneously the nitrogen rich graphene sheets, as verified with EDS mapping (Fig. 4b-e). HR-XPS further confirmed the presence and oxidation state of the $\mathrm{Pt}^{\mathrm{II}}$ ions, with the two spin-orbit split $4 \mathrm{f}$ components appearing at electron binding energies (EBE) of 73.1 and $76.5 \mathrm{eV}$. According to AAS analysis, the Pt content on g-CN/Pt was particularly high (3.7 wt \%), surpassing the recently reported SAC where $3 \mathrm{wt} \% \mathrm{Pt}$ loading on ceria was achieved [67]. No Pt was detectable in a control analysis of the as synthesized g-CN. Therefore, $\mathrm{g}-\mathrm{CN}$ acted as a very effective 2D-ligand for trapping and sustaining single Pt ions over the out-of-plane functionalized graphene.

\section{Conclusions}

In conclusion, we addressed the anchoring of single-platinumadatom on functionalized graphene. Since Pt adatoms easily diffuse on pristine graphene, which may lead to undesirable aggregation and nanoparticles formation, we explored graphene derivatives bearing $-\mathrm{F},-\mathrm{OH},-\mathrm{CN}$ and $-\mathrm{H}$ functional groups as possible means of anchoring single Pt atoms and preventing their diffusion. Whereas the Pt adatom cleaved the bonds of $-\mathrm{F},-\mathrm{OH}$, and $-\mathrm{H}$ to graphene leading to the formation of graphene-Pt-F $(-\mathrm{OH}$, or $-\mathrm{H})$ complexes, the $-\mathrm{CN}$ group remained bound to graphene lattice and anchored the single-platinum-adatom with high energy barrier against -CN-Pt migration. The anchoring of Pt adatoms on $\mathrm{g}-\mathrm{CN}$ provide a scaffold for SACs which are considered as one of the holy grails in catalysis as they enable the best use of rare and rather expensive noble metals. As cyanographene was experimentally prepared in 2017 , the $\mathrm{g}-\mathrm{CN}$ material seems to be very promising as an anchor for metals, as recently experimentally demonstrated with the synthesis of SACs based on $\mathrm{Cu}$ and Fe ions [21], but also in the present work with the effective immobilization of Pt ions enabling plethora of catalytic reactions which could be examined in future.

\section{Declaration of Competing Interest}

The authors declare no competing financial interests.

\section{Acknowledgements}

The authors gratefully acknowledge the support of the Ministry of Education, Youth and Sports of the Czech Republic under Project No. LO1305, the Operational Programme for Research, Development and Education of the European Regional Development Fund (Project No. CZ.02.1.01/0.0/0.0/16_019/0000754), the support from the Internal Student Grant Agency of the Palacký University in Olomouc, Czech Republic (IGA_PrF_2019_031) and the Spanish MCIU (RTI2018 - 101394 - BI00/MCIU/AEI/FEDER, UE). E. F. thanks MINECO La Caixa Severo Ochoa for a predoctoral grant through Severo Ochoa Excellence Accreditation 20142018 (SEV 2013 0319). M. O. acknowledges the ERC grant (683024) from the EU Horizon 2020 Research and Innovation Programme. The authors gratefully acknowledge BSC-RES for providing generous computational resources. Jana Stráská and Dr. Klára Čépe for the electron microscopy analysis, Jan Kolařik for AAS measurements, Ondřej Tomanec for HR-TEM, and Tomáš Steklý for synthesis of g-CN are gratefully acknowledged.

\section{References}

[1] K.S. Novoselov, A.K. Geim, S.V. Morozov, D. Jiang, Y. Zhang, S.V. Dubonos, I.V. Grigorieva, A.A. Firsov, Electric field effect in atomically thin carbon films, Science 306 (2004) 666-669, http://dx.doi.org/10.1126/science.1102896.

[2] K.S. Novoselov, A.K. Geim, S.V. Morozov, D. Jiang, M.I. Katsnelson, S.V. Grigorieva, S.V. Dubonos, A.A. Firsov, Two-dimensional gas of massless dirac fermions in graphene, Nature 438 (2005) 197-200, http://dx.doi.org/10.1038/ nature04233.

[3] K. Novoselov, Mind the gap, Nat. Mater. 6 (2007) 720-721, http://dx.doi.org/ $10.1038 /$ nmat2006.

[4] A.K. Geim, K.S. Novoselov, The rise of graphene, Nat. Mater. 6 (2007) 183-191, http://dx.doi.org/10.1038/nmat1849.

[5] V. Georgakilas, J.A. Perman, J. Tuček, R. Zbořil, Broad family of carbon nanoallotropes: classification, chemistry, and applications of fullerenes, carbon dots, nanotubes, graphene, Nanodiamonds, and combined superstructures, Chem. Rev. 115 (2015) 4744-4822, http://dx.doi.org/10. 1021/cr500304f

[6] A.H. Castro-Neto, F. Guinea, N.M.R. Peres, K.S. Novoselov, A.K. Geim, The electronic properties of graphene, Rev. Mod. Phys. 81 (2009) 109-162, http:// dx.doi.org/10.1103/RevModPhys.81.109.

[7] P. Błoński, Z. Łodziana, First-principles study of $\mathrm{LiBH}_{4}$ nanoclusters interaction with models of porous carbon and silica scaffolds, Int. J. Hydrogen. Energ. 39 (2014) 9848-9853, http://dx.doi.org/10.1016/j.ijhydene.2014.03.264.

[8] H. Wang, T. Maiyalagan, X. Wang. Review on recent progress in nitrogen-doped graphene: synthesis, characterization, and its potential applications, ACS Catal. 2 (2012) 781-794, http://dx.doi.org/10.1021/ cs200652y.

[9] P. Błoński, J. Tuček, Z. Sofer, V. Mazánek, M. Petr, M. Pumera, M. Otyepka, R. Zbořil, Doping with graphitic nitrogen triggers ferromagnetism in graphene, J. Am. Chem. Soc. 139 (2017) 3171-3180, http://dx.doi.org/10.1021/jacs. 6b12934.

[10] C. Lee, X. Wei, J.W. Kysar, J. Hone, Measurement of the Elastic Properties and Intrinsic Strength of Monolayer Graphene, Science 321 (2008) 385-388, http://dx.doi.org/10.1126/science.1157996.

[11] M.J. Allen, V.C. Tung, R.B. Kaner, Honeycomb carbon: a review of graphene, Chem. Rev. 110 (2010) 132-145, http://dx.doi.org/10.1021/cr900070d.

[12] W. Han, R.K. Kawakami, M. Gmitra, J. Fabian, Graphene spintronics, Nat. Nanotechnol. 9 (2014) 794-807, http://dx.doi.org/10.1038/nnano.2014.214.

[13] Z. Liu, Q. Liu, Y. Huang, Y. Ma, S. Yin, X. Zhang, W. Sun, Y. Chen, Organic photovoltaic devices based on a novel acceptor material, Graphene Adv. Mater. 20 (2008) 3924-3930, http://dx.doi.org/10.1002/adma.200800366.

[14] M.D. Stoller, S. Park, Y. Zhu, J. An, R.S. Ruoff, Graphene-based ultracapacitors, Nano Lett. 8 (2008) 3498-3502, http://dx.doi.org/10.1021/nl802558y.

[15] V. Urbanová, K. Holá, A.B. Bourlinos, K. Čépe, A. Ambrosi, A.H. Loo, M. Pumera, F. Karlický, M. Otyepka, R. Zbořil, Thiofluorographene-hydrophilic graphene derivative with semiconducting and genosensing properties, Adv. Mater. 27 (2015) 2305-2310, http://dx.doi.org/10.1002/adma.201500094.

[16] J. Tuček, K. Holá, B. Bourlinos, P. Błoński, A. Bakandritsos, J. Ugoloti, M. Dubecký, F. Karlický, V. Ranc, K. Čépe, M. Otyepka, R. Zbořil, Room temperature organic magnets derived from $\mathrm{sp}^{3}$ functionalized graphene, Nat. Commun. 8 (1-12) (2017) 14525, http://dx.doi.org/10.1038/ncomms14525.

[17] A. Bakandritsos, P. Jakubec, M. Pykal, M. Otyepka, Covalently functionalized graphene as a supercapacitor electrode material, FlatChem 13 (2019) 25-33, http://dx.doi.org/10.1016/j.flatc.2018.12.004.

[18] G. Carchini, N. Almora-Barrios, G. Revilla-López, L. Bellarosa, R. García-Muelas, M. García-Melchor, S. Pogodin, P. Błoński, N. López, How theoretical simulations can address the structure and activity of nanoparticles, Top. Catal. 56 (2013) 1262-1272, http://dx.doi.org/10.1007/s11244-013-0093-3.

[19] J.M. Thomas, The enduring relevance and academic fascination of catalysis, Nat. Catal. 1 (2018) 2-5, http://dx.doi.org/10.1038/s41929-017-0014-0.

[20] J.M. Thomas, W.J. Thomas, Principles and Practice of Heterogeneous Catalysis, Wiley - VCH, Weinheim, Germany, 2015.

[21] A. Bakandritsos, R.G. Kadam, P. Kumar, G. Zoppellaro, M. Medved', J. Tuček, T Montini, O. Tomanec, P. Andrýsková, B. Drahoš, R.S. Varma, M. Otyepka, M.B. Gawande, P. Fornasiero, R. Zbořil, Mixed-Valence Single-Atom Catalyst 
Derived from Functionalized Graphene, Adv. Mater. 31 (1-9) (2019) 1900323 http://dx.doi.org/10.1002/adma.201900323.

[22] M. Monai, M. Melchionna, P. Fornasiero, From metal to metal-free catalysts route to sustainable chemistry, J. Adv. Catal. Sci. Technol. 63 (2018) 1-73. http://dx.doi.org/10.1016/bs.acat.2018.10.001.

[23] H. Fei, J. Dong, Y. Feng, C.S. Allen, C. Wan, B. Volosskiy, M. Li, Z. Zhao, Y. Wang, H. Sun, P. An, W. Chen, Z. Guo, C. Lee, D. Chen, I. Shakir, M. Liu, T. Hu, Y. Li, A.I. Kirkland, X. Duan, Y. Huang, General synthesis and definitive structural identification of MN4C4 single-atom catalysts with tunable electrocatalytic activities, Nat. Catal. 1 (2018) 63-72, http://dx.doi.org/10.1038/s41929-017 0008-y.

[24] L. Yin, J. Liebscher, Carbon-Carbon coupling reactions catalyzed by heterogeneous palladium catalysts, Chem. Rev. 107 (2007) 133-173, http:// dx.doi.org/10.1021/cr0505674.

[25] J. Liu, Catalysis by supported single metal atoms, ACS Catal. 7 (2017) 34-59, http://dx.doi.org/10.1021/acscatal.6b01534.

[26] S. Wei, A. Li, J.C. Liu, Z. Li, W. Chen, Y. Gong, Q. Zhang, W.C. Cheong, Y. Wang, L. Zheng, H. Xiao, C. Chen, D. Wang, Q. Peng, L. Gu, X. Han, J. Li, Y. Li, Direct observation of noble metal nanoparticles transforming to thermally stable single atoms, Nat. Nanotechnol. 13 (2018) 856-861, http://dx.doi.org/10. 1038/s41565-018-0197-9.

[27] Z. Chen, E. Vorobyeva, S. Mitchell, E. Fako, M.A. Ortuño, N. López, S.M. Collins, P.A. Midgley, S. Richard, G. Vilé, J. Pérez-Ramírez, A heterogeneous single-atom palladium catalyst surpassing homogeneous systems for suzuki coupling, Nat. Nanotechnol. 13 (2018) 702-707, http://dx.doi.org/10.1038/ s41565-018-0167-2.

[28] Z. Chen, S. Mitchell, E. Vorobyeva, R.K. Leary, R. Hauert, T. Furnival, Q.M. Ramasse, J.M. Thomas, P.A. Midgley, D. Dontsova, M. Antonietti, S. Pogodin, N. López, J.A. Pérez- Ramírez, Stabilization of single metal atoms on graphitic carbon nitride, Adv. Funct. Mater. 27 (2017) 1-12, http://dx.doi.org/10.1002/ adfm.201605785.

[29] G. Vilé, D. Albani, M. Nachtegaal, Z. Chen, D. Dontsova, M. Antonietti, N. López, J.A. Pérez-Ramírez, Stable single-site palladium catalyst for hydrogenations, Angew. Chem. 54 (2015) 11265-11269, http://dx.doi.org/10.1002/anie. 201505073.

[30] A. Zitolo, V. Goellner, V. Armel, M. Sougrati, T. Mineva, L. Stievano, E. Fonda, F. Jaouen, Identification of catalytic sites for oxygen reduction in Iron- and nitrogen-doped graphene materials, Nat. Mater. 14 (2015) 937-942, http:// dx.doi.org/10.1038/nmat4367.

[31] S. Vajda, M.J. Pellin, J.P. Greeley, C.L. Marshall, L.A. Curtiss, G.A. Ballentine, J.W. Elam, S. Catillon-Mucherie, P.C. Redfern, F. Mehmood, P. Zapol, Subnanometre platinum clusters as highly active and selective catalysts for the oxidative dehydrogenation of propane, Nat. Mater. 8 (2009) 213-216, http://dx.doi.org/ 10.1038/nmat2384.

[32] E. Fako, Z. Lodziana, N. López, Comparative single atom heterogeneous catalysts (SAHCs) on different platforms: a theoretical approach, Catal. Sci. Technol. 7 (2017) 4285-4293, http://dx.doi.org/10.1039/C7CY01136A.

[33] X. Zhou, J. Qiao, L. Yang, J.A. Zhang, Review of graphene-based nanostructural materials for both catalyst supports and metal-free catalysts in PEM fuel cell oxygen reduction reactions, Adv. Energy Mater. 4 (1-25) (2014) 1301523, http://dx.doi.org/10.1002/aenm.201301523.

[34] S. Sun, G. Zhang, N. Gauquelin, N. Chen, J. Zhou, S. Yang, W. Chen, X. Meng, D. Geng, M.N. Banis, R. Li, S. Ye, S. Knights, G.A. Botton, T.-K. Sham, X. Sun, Single-atom catalysis using Pt/Graphene achieved through atomic layer deposition, Sci. Rep. 3 (1-9) (2013) 1775, http://dx.doi.org/10.1038/ srep01775.

[35] I.A. Pasti, A. Jovanovič, A.S. Dobrota, S.V. Mentus, B. Johansson, N.V. Skorodumova, Atomic adsorption on pristine graphene along the periodic table of elements - from PBE to non-local functionals, Appl. Surf. Sci. 436 (2017) 433-440, http://dx.doi.org/10.1016/j.apsusc.2017.12.046.

[36] I.A. Pasti, A. Jovanovič, A.S. Dobrota, S.V. Mentus, N.V. Skorodumova, Atomic adsorption on graphene with a single vacancy: systematic DFT study through the periodic table of elements, Phys. Chem. Chem. Phys. 20 (2017) 858-865, http://dx.doi.org/10.1039/C7CP07542A.

[37] B. de la Torre, M. Švec, P. Hapala, J. Redondo, O. Krejčí, R. Lo, D. Manna, A. Sarmah, D. Nachtigallová, J. Tuček, P. Błoński, M. Otyepka, R. Zbořil, P. Hobza, P. Jelínek, Non-covalent control of spin-state in metal-organic complex by positioning on N-doped graphene, Nat. Commun. 9 (1-9) (2018) 2831, http:// dx.doi.org/10.1038/s41467-018-05163-y.

[38] M. Lefèvre, E. Proietti, F. Jaouen, J.-P. Dodelet, Iron-based catalysts with improved oxygen reduction activity in polymer electrolyte fuel cells, Science 324 (2009) 71-74, http://dx.doi.org/10.1126/science.1170051.

[39] N. Cheng, S. Stambula, D. Wang, M.N. Banis, J. Liu, A. Riese, B. Xiao, R. Li, T.K. Sham, L.M. Liu, G.A. Botton, X. Sun, Platinum single-atom and cluster catalysis of the hydrogen evolution reaction, Nat. Commun. 7 (1-9) (2016) 13638 , http://dx.doi.org/10.1038/ncomms13638.

[40] X. Li, P. Cui, W. Zhong, J. Li, X. Wang, Z. Wang, J. Jiang, Graphitic carbon nitride supported single-atom catalysts for efficient oxygen evolution reaction, ChemComm. 52 (2016) 13233-13236, http://dx.doi.org/10.1039/ C6CC07049C.

[41] R. Zbořil, F. Karlický, A.B. Bourlinos, T.A. Steriotis, A.K. Stubos, V. Georgakilas, K. Safarova, D. Jancik, C. Trapalis, M. Otyepka, Graphene fluoride: a stable stoichiometric graphene derivative and its chemical conversion to graphene, Small 6 (2010) 2885-2891, http://dx.doi.org/10.1002/smll.201001401.
[42] Y. Zhu, S. Murali, W. Cai, X. Li, J.W. Suk, J.R. Potts, R.S. Ruoff, Graphene and graphene oxide: synthesis, properties, and applications, Adv. Mater. 22 (2010) 3906-3924, http://dx.doi.org/10.1002/adma.201001068.

[43] A. Bakandritsos, M. Pykal, P. Błoński, P. Jakubec, D.D. Chronopoulos, K. Poláková, V. Georgakilas, K. Čépe, O. Tomanec, V. Ranc, A.B. Bourlinos, R. Zbořil, M. Otyepka, Cyanographene and graphene acid: emerging derivatives enabling high-yield and selective functionalization of graphene, ACS Nano 11 (2017) 2982-2991, http://dx.doi.org/10.1021/acsnano.6b08449.

[44] J.O. Sofo, A.S. Chaudhari, G.D. Barber, Graphane: a two-dimensional hydrocarbon, Phys. Rev. B 75 (1-4) (2007) 153401, http://dx.doi.org/10.1103/ PhysRevB.75.153401.

[45] G. Kresse, J. Furthmüller, Efficiency of ab-initio total energy calculations for metals and semiconductors using a plane-wave basis set, Comp. Mater. Sci. 6 (1996) 15-50, http://dx.doi.org/10.1016/0927-0256(96)00008-0.

[46] G. Kresse, J. Furthmüller, Efficient iterative schemes for ab initio total-energy calculations using a plane-wave basis set, Phys. Rev. B 54 (1996) 11169-11186, http://dx.doi.org/10.1103/PhysRevB.54.11169.

[47] J.P. Perdew, K. Burke, M. Ernzerhof, Generalized gradient approximation made simple, Phys. Rev. Lett. 77 (1996) 3865-3868, http://dx.doi.org/10. 1103/PhysRevLett.77.3865.

[48] P.E. Blöchl, Projector augmented-wave method, Phys. Rev. B 50 (1994) 17953-17979, http://dx.doi.org/10.1103/physrevb.50.17953.

[49] G. Kresse, D. Joubert, From Ultrasoft Pseudopotentials to the Projector Augmented-Wave Method, Phys. Rev. B 59 (1999) 11-19, http://dx.doi.org/ 10.1103/PhysRevB.59.1758

[50] S. Grimme, S. Ehrlich, L. Goerigk, Effect of the damping function in dispersion corrected density functional theory, J. Comput. Chem. 32 (2011) 1456-1465, http://dx.doi.org/10.1002/jcc.21759.

[51] S. Grimme, J. Antony, S. Ehrlich, H. Krieg, Consistent and accurate ab initio parametrization of density functional dispersion correction (DFT-D) for the 94 elements H-Pu, J. Chem. Phys. 132 (1-19) (2010) 154104, http://dx.doi.org/10. $1063 / 1.3382344$

[52] G. Makov, M.C. Payne, Periodic boundary consitions in ab initio calculations, Phys. Rev. B 51 (1994) 4014-4022, http://dx.doi.org/10.1103/physrevb.51. 4014.

[53] G.A. Henkelman, B.P. Uberuaga, H. Jónsson, Climbing image nudged elastic band method for finding saddle points and minimum energy paths and minimum energy paths, J. Chem. Phys. 113 (2010) 9901-9904, http://dx.doi. org/10.1063/1.1329672.

[54] G. Henkelman, H. Jónsson, Improved tangent estimate in the nudged elastic band method for finding minimum energy paths and saddle points minimum energy paths and saddle points, J. Chem. Phys. 113 (2000) 9978-9985, http:// dx.doi.org/10.1063/1.1323224.

[55] E. Sanville, S.D. Kenny, R. Smith, G. Henkelman, Improved grid-based algorithm for bader charge allocation, J. Comput. Chem. 28 (2007) 899-908, http://dx.doi.org/10.1002/jcc.20575.

[56] R. Heyrovska, The golden ratio, ionic and atomic radii and bond lengths, Mol. Phys. 103 (2007) 877-882, http://dx.doi.org/10.1080/ 00268970412331333591

[57] M.J. Frisch, G.W. Trucks, H.B. Schlegel, G.E. Scuseria, M.A. Robb, J.R. Cheeseman, G. Scalmani, V. Barone, B. Mennucci, G.A. Petersson, H. Nakatsuji, M. Caricato, X. Li, H.P. Hratchian, A.F. Izmaylov, J. Bloino, G. Zheng, J.L. Sonnenberg, M. Hada, M. Ehara, K. Toyota, R. Fukuda, J. Hasegawa, M. Ishida, T. Nakajima, Y. Honda, O. Kitao, H. Nakai, T. Vreven, J.A. Montgomery, J.E. Peralta, F. Ogliaro, M. Bearpark, J.J. Heyd, E. Brothers, K.N. Kudin, V.N. Staroverov, R. Kobayashi, J. Normand, K. Raghavachari, A. Rendell, J.C. Burant, S.S. Iyengar, J. Tomasi, M. Cossi, N. Rega, J.M. Millam, M. Klene, J.E. Knox, J.B. Cross, V. Bakken, C. Adamo, J. Jaramillo, R. Gomperts, R.E. Stratmann, O. Yazyev, A.J. Austin, R. Cammi, C. Pomelli, J.W. Ochterski, R.L. Martin, K. Morokuma, V.G. Zakrzewski, G.A. Voth, P. Salvador, J.J. Dannenberg, S. Dapprich, A.D. Daniels, O. Farkas, J.B. Foresman, J.V. Ortiz, J. Cioslowski, D.J. Fox, Gaussian 09, Revision D.01, Wallingford CT, 2009.

[58] J.P. Perdew, K. Burke, M. Ernzerhof, Errata: generalized gradient approximation made simple, Phys. Rev. Lett. 78 (1997) 1396

[59] M. Álvarez-Moreno, C. de Graaf, N. López, F. Maseras, J.M. Poblet, C. Bo, Managing the computational chemistry big data problem: the ioChem-BD platform, J. Chem. Inf. Model. 55 (2015) 95-103, http://dx.doi.org/10.1021/ ci500593j.

[60] Y. Li, W. Gao, L. Ci, C. Wang, P.M. Ajayan, Catalytic performance of Pt nanoparticles on reduced graphene oxide for methanol electro-oxidation, Carbon 48 (2009) 1124-1130, http://dx.doi.org/10.1016/j.carbon.2009.11.034.

[61] Z. Liu, X. Duan, H. Cheng, J. Zhou, X. Zhou, Synthesis of Platinum/Graphene composites by a polyol method: the role of graphite oxide precursor surface chemistry, Carbon 89 (2015) 93-101, http://dx.doi.org/10.1016/j.carbon. 2015.03.022.

[62] Y.H. Cheong, M.Z.M. Nasir, A. Bakandritsos, M. Pykal, P. Jakubec, R. Zbořil, M. Otyepka, M. Pumera, Cyanographene and Graphene Acid: The Functional Group of Graphene Derivative Determines the Application in Electrochemical Sensing and Capacitors, ChemElectroChem. 6 (2019) 229-234, http://dx.doi. org/10.1002/celc. 201800675.

[63] O.V. Yazyev, L. Helm, Defect-induced magnetism in graphene, Phys. Rev. B 75 (1-5) (2007) 125408, http://dx.doi.org/10.1103/PhysRevB.75.125408.

[64] M. Wu, E.-Z. Liu, J.Z. Jiang, Magnetic behavior of graphene absorbed with N, O and F atoms: a first-principles study, Appl. Phys. Lett. 93 (1-3) (2008) 082504 , http://dx.doi.org/10.1063/1.2973209. 
[65] T.-T. Jia, B.-Z. Sun, H.-X. Lin, Y. Li, W.-K. Chen, Bonding of hydroxyl and epoxy roups on graphene: insights from density functional calculations, Chinese J. Struct. Chem. 32 (2013) 1475-1484.

[66] I.V. Lightcap, T.H. Kosel, P.V. Kamat, Anchoring semiconductor and metal nanoparticles on a two-dimensional catalyst mat. Storing and shuttling electrons with reduced graphene oxide, Nano Lett. 10 (2010) 577-583, http:// dx.doi.org/10.1021/n19035109.
[67] D. Kunwar, S. Zhou, A. DeLaRiva, E.J. Peterson, H. Xiong, X.I.

Pereira-Hernández, S.C. Purdy, R. ter Veen, H.H. Brongersma, J.T. Miller, H. Hashiguchi, L. Kovarik, S. Lin, H. Guo, Y. Wang, A.K. Datye, Stabilizing high metal loadings of thermally stable platinum single atoms on an industrial catalyst support, ACS Catal. 9 (2019) 3978-3990, http://dx.doi.org/10.1021/ acscatal.8b04885. 